\title{
Dynamic turn-on behavior of organic light-emitting devices with different work function cathode metals under fast pulse excitation
}

\author{
Musubu Ichikawa, ${ }^{\text {a) }}$ Junko Amagai, Yasuhiro Horiba, Toshiki Koyama, \\ and Yoshio Taniguchi \\ Department of Functional Polymer Science, Faculty of Textile Science and Technology, Shinshu University, \\ 3-15-1 Tokita, Ueda City, Nagano 386-8567, Japan
}

(Received 28 July 2003; accepted 2 October 2003)

\begin{abstract}
We investigate the electroluminescence (EL) response of organic light-emitting diodes (OLEDs) with different metal cathodes and device-area dependence. Moreover, we formulate the EL delay time, which is an indicator of the EL response. The EL response is fundamentally governed by the device area, i.e., the capacitance of the device, and this for the OLEDs with low work function metals as a cathode is fast. According to the formulation, we estimate electron mobility in an organic bilayer and the threshold electric fields for electron injection under fast-pulse excitation. It is consequently demonstrated that electron mobility in the bilayer showed mixed mobility characteristics for each layer, and the threshold electric field became smaller with a decrease in the work function of cathode metals. The low work function metal cathode device is identified as a high-speed EL response device. (C) 2003 American Institute of Physics.
\end{abstract}

[DOI: $10.1063 / 1.1628835]$

\section{INTRODUCTION}

Impressive scientific and technological advances have been achieved in the area of organic light-emitting diodes (OLEDs) in this decade. This has been driven by the potential applications for devices in a large variety of display technologies. In addition, fundamental research to gain a better understanding of behavior of charge carriers in organic semiconductors, for example, injection from electrodes, migration (transport), and the radiative carrier recombination, have also been motivated by prospects to improve device performance and invent a new organic device. Electroluminescence (EL) from OLEDs is generated by a recombination between holes and electrons, which are injected from an anode and cathode. Therefore, the dynamic behavior of EL of OLEDs under electrical fast pulse excitation provides important insights into the internal operation mechanisms [injection, transport (migration), and recombination of charges] of OLEDs and the device physics of organic semiconductors. ${ }^{1-11}$ For example, it is easy to imagine that the EL dynamics under fast pulse excitation conditions give valuable information about charge carrier injections into organic layers of OLEDs, charge transport in the layers of OLEDs, and so on. The transient response is also important in the context of possible applications for OLEDs in optical communications where their utility will ultimately be limited by the response of OLED light sources to high frequency modulation. ${ }^{12}$

In this article, we investigate the EL response and its device-area dependence of three organic layered OLED devices with different metal cathodes to obtain internal information about both charge injection from electrodes to organics and charge transport in organic multilayers, and also to

a) Author to whom correspondence should be addressed; electronic mail: musubu@giptc.shinshu-u.ac.jp understand and thus control limited processes for fast EL response. Although the turn-on time of EL response under pulsed excitation was frequently discussed in relation to the time to carrier transport time in single organic layers, ${ }^{1,9,13-16}$ there has been no report on charge transport times in organic multilayers as revealed by a transient EL response analysis.

\section{EXPERIMENT}

Sample devices were prepared by the following procedures. An indium-tin-oxide (ITO)-coated glass substrate was treated by $\mathrm{O}_{2}$ plasma for $5 \mathrm{~min}$ in advance. Organic layers and cathode metals were sequentially deposited on the ITO substrate in a vacuum $\left(3 \times 10^{-4} \mathrm{~Pa}\right)$. Device structures and chemicals used in this study are shown in Fig. 1. To change the electron injection barrier from a cathode to an organic layer, two kinds of metal, $\mathrm{Ca}$ and $\mathrm{Mg}: \mathrm{Ag}$ (9:1 in volume), were used as cathodes. In the case of the Ca cathode, a 300-nm-thick Al layer was successively deposited on a 10-nm-thick active metal layer to protect the active metal from the atmosphere. With the other cathode cases, a 300nm-thick metal cathode was deposited. The device area, which was measured with a Keyence VH-8000 digital optical microscope, was varied from approximately 0.25 to $6 \mathrm{~mm}^{2}$ by changing only the cathode area. Thus, the size and thickness of the ITO, which has high resistivity compared with cathode metals, were fixed in all devices, meaning that the external series resistance (wiring resistance) of the device remained unchanged when the area varied. The transient EL wave forms were measured with a nanosecond apparatus, of which the schematic details are shown in Fig. 2. A fast electric pulse generator (Model 2000D, Picosecond Pulse Lab.), generating a rectangular voltage pulse of $210 \mathrm{~ns}$ width with a $500 \mathrm{ps}$ rise time at a $10 \mathrm{~Hz}$ repetition rate was used to drive the device through an electric power attenuator (TRA-10B, 


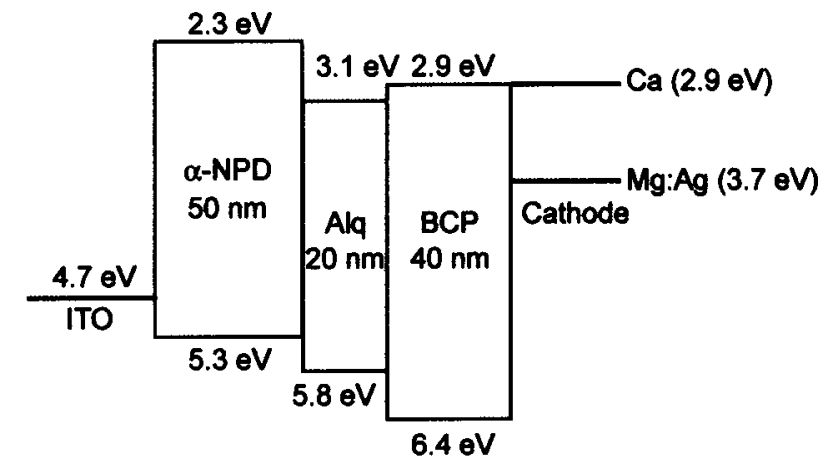



a-NPD



Alq



$\mathrm{BCP}$
FIG. 1. Schematic illustration of device structure, work functions of the cathode metals used, and structures and notations of chemicals used.

Tamagawa Electronics). Light emitted from the sample OLED is detected by a photomultiplier (R7400U, Hamamatsu Photonics) through an optical fiber bundle and a monochromator. The signal from the photomultiplier is recorded using an oscilloscope (TDS-680C, Sony Tektronics) via an ultrafast preamplifier with a fixed gain of $36 \mathrm{~dB}$ (C5594, Hamamatsu Photonics). The time delay of the apparatus, including the electric delays in the apparatus itself and in the cables and so on, was experimentally determined for each measurement so that the time axis could be adjusted for the delay. Figure 1 shows a schematic illustration of the device structure along with the work function of the metals used.

\section{RESULTS AND DISCUSSION}

\section{A. Transient EL behavior of OLEDs}

Figure 3 shows the transient EL wave forms observed from the OLED devices, of which the device structure is ITO/NPD/Alq $(20 \mathrm{~nm}) / \mathrm{BCP}(40 \mathrm{~nm}) / \mathrm{Ca}$ or $\mathrm{Mg}: \mathrm{Ag}$ cathode, at an applied pulse voltage of $36 \mathrm{~V}$, with an emitting area of $4 \mathrm{~mm}^{2}$. As shown in the figure, the starting times of the transient EL wave forms (indicated by the arrows) was delayed from the pulse was applied (at point 0 on the time axis). The time lag between pulse application and the transient EL wave form is defined here as the "EL delay time."



FIG. 2. Schematic diagram of measurement system for nanosecond transient EL: (MC) monochromator and (PM) photomultiplier.

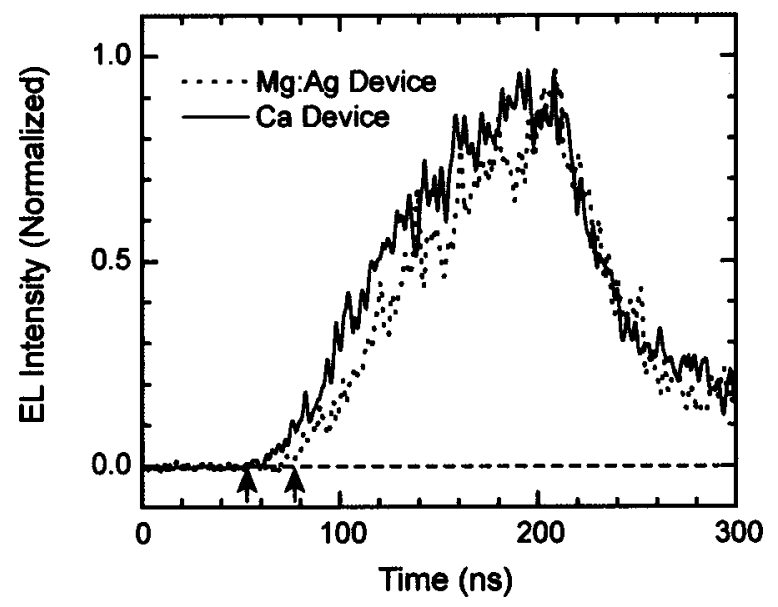

FIG. 3. Fast transient EL wave forms observed from the OLED devices with different metal cathodes ( $\mathrm{Ca}$ and $\mathrm{Mg}: \mathrm{Ag}$ ) at applied pulse voltage of $36 \mathrm{~V}$, with emitting area of $4 \mathrm{~mm}^{2}$.

As is also shown in Fig. 3, the EL delay time depended on the cathode metal. The delay times of the $\mathrm{Ca}$ and $\mathrm{Mg}: \mathrm{Ag}$ cathode devices were, respectively, 52 and $77 \mathrm{~ns}$.

Figure 4 shows the device-area dependence of the EL delay times of OLEDs using each of the various kinds of cathodes at an applied voltage of $32 \mathrm{~V}$. As shown in the figure, the EL delay times depend on metal species of the cathodes, and furthermore, the delay times proportionally increase with an increase in the device area of all of the cathodes. This proportional dependence indicates that the EL response of OLEDs is fundamentally governed by the electrostatic capacitance of the devices. This capacitance originates from thin and large device structures of OLEDs. OLEDs are virtually assumed to be capacitors consisting of a cathode, an anode, and organic thin layers (insulators). The external series resistance of OLED devices also affects the EL delay time through a well-known parameter, the time constant $C R$ (the product of the capacitance and the series external resistance of the OLED device). However, as described in Sec. II, the external series resistance of OLED remains unchanged for all devices in this study (the differ-

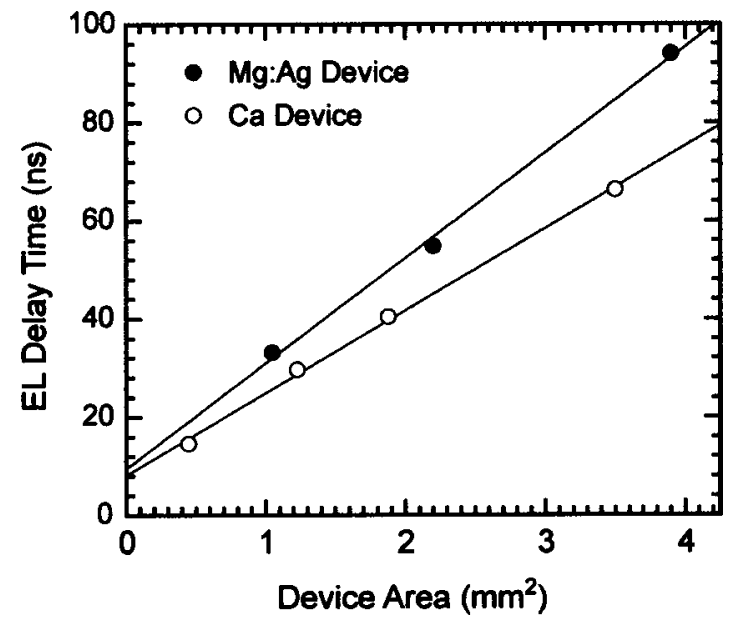

FIG. 4. Device area dependence of EL delay times for OLEDs using various cathode metals at applied voltage of $32 \mathrm{~V}$. 
ence of the resistance between the $\mathrm{Ca}$ and $\mathrm{Mg}: \mathrm{Ag}$ devices is very small, because resistivity of the metals is significantly small compared with that of ITO). Therefore, it is safely concluded that the device area dependence shown in Fig. 4 is caused by the capacitance not the external series resistance of the OLED.

As shown in Fig. 4, the slopes of the device area dependence vary by changing the cathode metals. The slope will be related to the specific time constant (time constant per unit area) of the system involving the OLED and the driver because of the above-mentioned proportional dependence. This specific time constant consists of the specific capacitance (capacitance per unit area of device) of the OLED, the external series resistance of OLEDs (main component of the resistance is caused by ITO), and the output impedance of the electric driver. Because the external series resistance and the output impedance are constant, the specific capacitance of the device is a key factor. In this study the device structure, concretely, the organic materials and thickness of each organic layer, is unchanged excluding cathode metals, and therefore the specific capacitance, in which OLEDs are regarded as a thin layer capacitor, is essentially unchanged, and of course, resistivity of organic layers is also unchanged. However, the EL delay time depends on cathode metal species. We will now try to formulate dynamic behavior of transient EL wave forms, especially of the EL delay time.

\section{B. Formulation of EL delay}

First, it is assumed that the EL delay time can be divided into two components: (1) the charge injection time, which means the time taken to charge the OLED to the threshold voltage $\left(V_{\text {th }}\right)$ for the carrier injection (from the electrode) of the second arrived carrier species at the emission zone and (2) the charge transport time of the second arrived carrier from the electrode to the emission zone. In this case, the EL delay time, $t_{\text {delay }}$, is equal to the sum of the injection delay, $t_{\text {inj }}$, and the transport delay, $t_{\text {trans }}$. Thus, the EL delay time is exhibited by

$$
t_{\text {delay }}=t_{\text {inj }}+t_{\text {trans }} .
$$

Assuming the equivalent circuit of the OLED device to be a series-connected circuit of a resistor and a capacitor (consisting of the anode and cathode of the OLED), the voltage difference between the anode and cathode at a time $(t)$ is expressed as a simple exponential growth function [1 $-\exp (-t / \tau)]$. Thus, the injection delay $t_{\text {inj }}$ is expressed by Eq. (2)

$$
t_{\mathrm{inj}}=-\tau \ln \left(1-\frac{E_{\mathrm{th}}}{E_{0}}\right),
$$

where $\tau$ is the time constant of the OLED device with the drive system, $E_{\text {th }}$ is the threshold electric field strength for charge injection, and $E_{0}$ is the electric field strength of the applied pulse voltage. For the sake of simplicity in the formulations below described $E_{\text {th }}$ and $E_{0}$, which are defined here as the ratio of the voltages ( $V_{\text {th }}$ and the pulse voltage) and the thickness of the organic layer of OLEDs, are used in Eq. (2). Assuming $E_{\mathrm{th}} \ll E_{0}, \mathrm{Eq}(2)$ is derived as follows:

$$
t_{\mathrm{inj}} \approx \tau \frac{E_{\mathrm{th}}}{E_{0}}=\tau_{i} S \frac{E_{\mathrm{th}}}{E_{0}}=\left(R+R_{0}\right) C_{i} S \frac{E_{\mathrm{th}}}{E_{0}},
$$

where $\tau_{i}$ and $S$ are the specific time constant per unit area of the time constant $\tau$ and the area of the OLED, respectively, and $R, R_{0}$, and $C_{i}$ represent the series resistance of OLEDs mainly caused by ITO, the additional series resistance of the drive system (50 $\Omega$, see Fig. 2), and the specific capacitance per unit area of the OLED assumed as a capacitor. From Eq (3), it is confirmed that the injection delay is proportional to the device area.

On the other hand, the charge transport time can be obtained as the solution to the following equation:

$$
d=\int_{t_{\mathrm{inj}}}^{t_{\mathrm{inj}}+t_{\text {trans }}} \mu E d t,
$$

where $d, \mu$, and $E$ are the run distance of the second arrived charge carrier species from electrode to the recombination zone, the mobility of the second arrived charge, and the electric field strength at the inside of OLEDs, respectively. Here, we suppose the following conditions: (1) the electric field strength inside the device is uniform and (2) carrier mobility is constant while injected carriers arrive at the recombination zone. Therefore, Eq. (4) can be reduced to the following equation:

$$
d=\mu E_{0}\left\{t_{\text {trans }}-\tau\left(1-\frac{E_{\text {th }}}{E_{0}}\right)\left[1-\exp \left(-\frac{t_{\text {trans }}}{\tau}\right)\right]\right\} .
$$

Here, assuming $t_{\text {trans }} \ll \tau$, we can obtain the transport time $t_{\text {trans }}$ as the solution of Eq. (5)

$$
t_{\text {trans }} \approx \frac{d}{\mu E_{\text {th }}} .
$$

Consequently, the EL delay time is approximately expressed as Eq. (7) from Eqs. (1), (3), and (6)

$$
t_{\text {delay }}=\left(R+R_{0}\right) C_{i} S \frac{E_{\text {th }}}{E_{0}}+\frac{d}{\mu E_{\mathrm{th}}} .
$$

Equation (7) qualitatively explains the characteristics of Fig. 4 of the proportional device area dependence and the fast response of the Ca cathode device because the OLED with lower work function cathode metal has smaller $E_{\mathrm{th}}$.

\section{Analysis of experimental results}

According to Eq. (7), the slope and the $y$ intercept of the linear fitting of Fig. 4 can be related to the threshold electric field strength $E_{\text {th }}$ and the mobility of the second arrived charge carrier species $\mu$. Figure 5 shows the calculated mobilities of the second arrived carrier species versus the square root of the applied electric fields, which is calculated as a ratio of the applied pulse voltage and the total thickness of the OLED organic layers. In the above calculation, $(R$ $\left.+R_{0}\right) C_{i}=\tau_{i}$ was set to be $1.6 \mathrm{~ns} / \mathrm{mm}^{2}$, which was confirmed by a measurement of the voltage rise time of the same OLED at a small applied voltage where no charge injection occurred (at $0.7 \mathrm{~V}$ ). ${ }^{17}$ Distance $d$ was temporarily set to be $60 \mathrm{~nm}$ (it was assumed that the recombination zone was located at the NPD/Alq interface and the second arrived carrier species was 


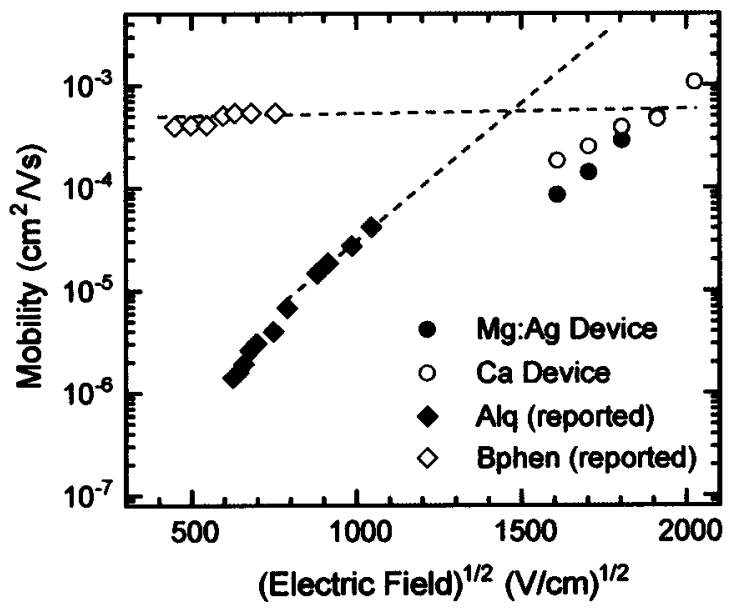

FIG. 5. Plots of calculated carrier mobility for the $\mathrm{Mg}: \mathrm{Ag}(\bullet)$ and $\mathrm{Ca}(\mathrm{O})$ cathode devices and reported electron mobility of Alq $(\diamond)$ and Bphen $(\diamond)$.

electron, see below). As shown in Fig. 5, the calculated mobilities for the different cathodes $\mathrm{Ca}$ and $\mathrm{Mg}: \mathrm{Ag}$ are close to each other, and their electric field dependence shows conventional characteristics for organic semiconductors, namely, $\mu$ $\propto \exp (\gamma \sqrt{E})$. It can be concluded that the device area dependence of the EL delay time can be consistently analyzed using Eq. (7).

In general, the electron mobility of organic semiconductors is much smaller than hole mobility. Therefore, the obtained carrier mobility can be related to electron mobility. Figure 5 also shows the electron mobility of $\mathrm{Alq}^{15}$ and bathophenanthroline (Bphen), ${ }^{18}$ which is a quite similar organic material to BCP. Compared with the electron mobilities of Alq and Bphen at the same electric field strength region using extrapolated straight lines for both materials, the obtained mobility shows characters of both materials. That is, mobility values are comparable to the extrapolated values of Bphen, and the slope of the mobility is close to that of Alq. In the OLEDs used in this study, electrons run in both Alq and BCP layers. It is quite consistent that the calculated mobility shows the behavior mentioned above. It can be safely concluded that we observed the charge transport behavior through the organic multilayer.

On the other hand, the threshold electric fields $E_{\text {th }}$ were calculated to be approximately $3.0 \pm 0.2 \mathrm{MV} / \mathrm{cm}$ for the $\mathrm{Ca}$ cathode device and 3.4 $\pm 0.2 \mathrm{MV} / \mathrm{cm}$ for the $\mathrm{Mg}: \mathrm{Ag}$ device. The threshold for the Ca device is smaller than that for the $\mathrm{Mg}: \mathrm{Ag}$ device. This fact is consistent with the difference in the electron injection barrier height estimated from the energy difference between the lowest unoccupied molecular orbital level of BCP and the work function of the cathode metal. As is seen in Fig. 1, the injection barrier for the $\mathrm{Ca}$ device is smaller than that for the $\mathrm{Mg}: \mathrm{Ag}$ device. Figure 6 exhibits the luminance-voltage characteristics of the $\mathrm{Ca}$ and $\mathrm{Mg}: \mathrm{Ag}$ devices and shows the turn-on voltage of the $\mathrm{Ca}$ cathode device is smaller than that of the $\mathrm{Mg}: \mathrm{Ag}$ device. The turn-on voltage, which is defined here as an applied voltage to get a luminance of $10 \mathrm{~cd} / \mathrm{m}^{2}$, is $12 \mathrm{~V}$ (namely, $11 \mathrm{MV} / \mathrm{cm}$ ) for the $\mathrm{Ca}$ device and $15.5 \mathrm{~V}(14 \mathrm{MV} / \mathrm{cm})$ for the $\mathrm{Mg}: \mathrm{Ag}$ device. This fact is qualitatively consistent with the relation-

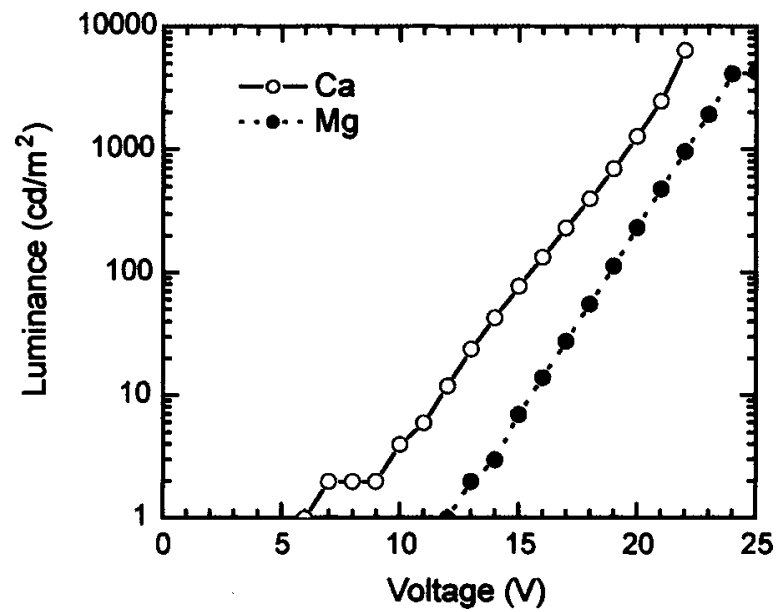

FIG. 6. Voltage-luminance characteristics of the $\mathrm{Mg}: \mathrm{Ag}$ (dotted line) and $\mathrm{Ca}$ (solid line) devices.

ship of the above-mentioned threshold electric field. However, there is no quantitative agreement. Even though this quantitative mismatch is induced by underestimation of specific time constant of OLEDs [see Eq. (7)], one of the reasons for the quantitative mismatch is probably the difference in charge injection behavior between dynamic excitation at very low duty by a single fast electric pulse and continuous dc excitation involving high-duty pulsed excitation, for example, the dynamic building up of space charges in organic layers, the orientational polarization of molecules, and so on. ${ }^{11,19,20}$ This is because the measured specific capacitance is close to the capacitance per unit area of the condenser with a relative permittivity of 3 (representative value of most organic solids) and insulator thickness of $110 \mathrm{~nm} .{ }^{17}$

\section{CONCLUSIONS}

We investigated the EL response of OLEDs with different metal cathodes and the device-area dependence. We formulated the EL delay time, which indicates the EL response. The EL response was fundamentally governed by the device area, i.e., the capacitance of the device. Moreover, the response of the OLEDs with low work function metals as a cathode was fast. Formulation of the EL delay time approximately reproduced the same behaviors, and the analysis by this formulation showed that the EL response of OLEDs under fast-pulse excitation was mainly controlled by electron injection and its transport. Accordingly, we estimated electron mobility in an organic bilayer consisting of Alq and BCP and the threshold electric field for electron injection under fast-pulse excitation. It was demonstrated that electron mobility in the bilayer showed mixed mobility characteristics for Alq and BCP, and the threshold electric field became smaller with a decrease in the work function of cathode metals. The low work function metal cathode device was identified as a high-speed EL response device.

\section{ACKNOWLEDGMENTS}

This work was supported by the Cooperative Link of Unique Science and Technology for Economy Revitalization 
of the Ministry of Education, Culture, Sports, Science and Technology. It was also supported by the 21 st century COE program, and a grant-in-aid for COE Research (Grant No. 10CE2003) from the Ministry of Education, Culture, Sports, Science, and Technology.

${ }^{1}$ C. Hosokawa, H. Tokailin, H. Higashi, and T. Kusumoto, Appl. Phys. Lett. 60, 1220 (1992).

${ }^{2}$ D. Braun, D. Moses, C. Zhang, and A. J. Heeger, Appl. Phys. Lett. 61, 3092 (1992).

${ }^{3}$ H. Vestweber, R. Sander, A. Greiner, W. Heitz, and R. F. Mahrt, Synth. Met. 64, 141 (1994).

${ }^{4}$ V. R. Nikitenko, V. I. Arkhipov, Y.-H. Tak, J. Pommerehne, H. Bassler, and H.-H. Horhold, J. Appl. Phys. 81, 7514 (1997).

${ }^{5}$ V. Savvate'ev, J. H. Friedl, L. Zou, J. Shinar, K. Christensen, W. Oldham, L. J. Rothberg, Z. Chen-Esterlit, and R. Kopelman, Appl. Phys. Lett. 76, 1501 (2000).

${ }^{6}$ K. Book, H. Bassler, V. R. Nikitenko, and A. Elschner, Synth. Met. 111112, 263 (2000).

${ }^{7}$ S. Das and A. J. Pal, Appl. Phys. Lett. 76, 1770 (2000).

${ }^{8}$ M. Ichikawa, R. Naitou, T. Koyama, and Y. Taniguchi, Jpn. J. Appl. Phys., Part 2 40, L1068 (2001).

${ }^{9}$ J. Wang, R. G. Sun, G. Yu, and A. J. Heeger, J. Appl. Phys. 91, 2417 (2002).
${ }^{10}$ M. Ichikawa, Y. Horiba, H. Nakatani, M. Yamada, T. Koyama, and Y. Taniguchi, Jpn. J. Appl. Phys., Part 1 41, 2252 (2002).

${ }^{11}$ L. Hassine, H. Bouchriha, J. Roussel, and J.-L. Fave, J. Appl. Phys. 91, 5170 (2002).

${ }^{12}$ H. Kajii et al., Jpn. J. Appl. Phys., Part 1 41, 2746 (2002).

${ }^{13}$ Y. Ohmori, C. Morishita, M. Uchida, and K. Yoshino, Jpn. J. Appl. Phys., Part 2 31, L568 (1992).

${ }^{14}$ C. Hosokawa, H. Tokailin, H. Higashi, and T. Kusumoto, Appl. Phys. Lett. 63, 1322 (1993).

${ }^{15}$ A. G. Muckl, S. Berleb, W. Brutting, and M. Schwoerer, Synth. Met. 111-112, 91 (2000).

${ }^{16}$ T. C. Wong, J. Kovac, C. S. Lee, L. S. Hung, and S. T. Lee, Chem. Phys. Lett. 334, 61 (2001).

${ }^{17} C_{i}$ measured by a frequency response analyzer (1260, Solatron, frequencies from $100 \mathrm{~Hz}-1 \mathrm{MHz}$ at an amplitude of $50 \mathrm{mV}$ without bias) was 222 $\mathrm{pF} / \mathrm{mm}^{2}$, and specific capacitance of a condenser with a relative permittivity of 3 and an insulator thickness of $110 \mathrm{~nm}$ was $241 \mathrm{pF} / \mathrm{mm}^{2}$. The measured $C_{i}$ is close to this value. This strongly confirms that it is consistent in our formulation to assume OLEDs as a capacitor at applied voltages under a threshold for charge injection.

${ }^{18}$ S. Naka, H. Okada, H. Onnagawa, and T. Tsutsui, Appl. Phys. Lett. 76, 197 (2000).

${ }^{19}$ L. Hassine, H. Bouchriha, J. Roussel, and J.-L. Fave, Appl. Phys. Lett. 78, 1053 (2001).

${ }^{20}$ S. Barth, P. Muller, H. Riel, P. F. Seidler, W. Riess, H. Vestweber, and H. Bassler, J. Appl. Phys. 89, 3711 (2001). 